\title{
Potentially inappropriate prescribing in Australian nursing home residents with advanced dementia: a sub-study of the IDEAL Study
}

Potentially inappropriate prescribing in advanced dementia

\section{Abstract}

Background Prescribing medications for nursing home residents with advanced dementia should focus on optimising function and comfort, reducing unnecessary harms and aligning care goals with a palliative approach.

Objective To estimate the proportion of Australian nursing home residents with advanced dementia receiving potentially inappropriate medications, identify those most commonly prescribed, and factors associated with their use.

Design Data were collected through retrospective audit of medication charts.

Setting/Subjects Two hundred and eighteen nursing home residents with advanced dementia from 20 nursing homes participating in a cluster randomised controlled trial of case conferencing (the IDEAL Study) from June 2013 to December 2014.

Measurements Inappropriate drug use was defined as medications classified as 'never appropriate' by the Palliative Excellence in Alzheimer Care Efforts program (PEACE) criteria. Generalized linear mixed models were used to identify variables predicting use of 'never' appropriate medications.

Results Over a quarter ( $n=65,30 \%$ ) of residents received at least one medication classed as 'never' appropriate, the most common being lipid-lowering agents $(n=38,17.4 \%)$, antiplatelet agents $(n=18$, $8.3 \%)$ and acetylcholinesterase inhibitors ( $n=16,7.3 \%)$. Residents who had been at the nursing home for $\leq 10$ months (odds ratio [OR] 5.60, 95\% confidence intervals [CI] 1.74-18.06), and 11 to 21 months (OR 5.41, Cl 1.67-17.75) had significantly greater odds of receiving a never appropriate medication compared to residents who had been at the nursing home for $>5$ years.

Conclusions Use of potentially inappropriate medications in Australian nursing home residents with advanced dementia is common. A greater understanding of the rationale that underpins prescribing of medications is required.

\section{Keywords}

Potentially inappropriate prescribing, polypharmacy, dementia, aged care, deprescribing 


\section{Introduction}

A palliative approach is best practice for people with advanced dementia. Their care and comfort should focus on minimising suffering. ${ }^{1,2}$ The role of medications in a palliative approach is primarily to relieve symptoms and are prescribed when adverse effects are outweighed by the likelihood of benefits that fall within the individual's life expectancy and no non-pharmacological alternatives are available ${ }^{3}$. Adverse effects associated with potentially inappropriate prescribing in older adults are associated with increased hospitalisation and mortality ${ }^{4,5}$ and involves extensive costs to healthcare systems. ${ }^{6,7}$ People with advanced dementia are particularly vulnerable to adverse drugs reactions as they undergo extreme physiological changes (in addition to those of normal ageing) that affect the way medications are metabolised in the body. ${ }^{8-10}$

Whilst there are several criteria available for assessing the appropriateness of prescribing in the elderly, ${ }^{11}$ these are not readily applicable to a palliative approach to prescribing in advanced dementia. ${ }^{12}$ A recent systematic review identified only one currently available dementia-specific criteria for identifying potentially inappropriate medications, which were developed through the Palliative Excellence in Alzheimer Care Efforts Program (PEACE) ${ }^{13}$ and classify medications as 'never', 'rarely', 'sometimes' or 'always' appropriate based on a Delphi process carried out with 12

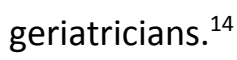

Six international studies have used these criteria to examine the prevalence of inappropriate prescribing in nursing home residents with advanced dementia and associated factors. ${ }^{15-20}$ These studies were undertaken in European countries, ${ }^{17-19}$ the USA ${ }^{15,} 16$ and Canada, ${ }^{20}$ and found that proportions of nursing home residents with advanced dementia receiving 'never' appropriate medications ranged from $3 \%{ }^{17}$ to $45 \%{ }^{20}$ of study populations. Lipid-lowering agents, ${ }^{15}, 16,18,20$ antiplatelet agents, ${ }^{17,}{ }^{18,} 20$ antihypertensives, ${ }^{17}$ and anti-dementia drugs, specifically acetylcholinesterase inhibitors ${ }^{15,16,18,20}$ and memantine, ${ }^{15}$ were found to be the most commonly prescribed 'never' appropriate medications. Predictors found to be associated with receiving 'never' appropriate medications included male gender, ${ }^{16}$ a higher functional status, ${ }^{16}$ earlier stage dementia, ${ }^{16,20}$ a shorter length of stay at the nursing home, ${ }^{16}$ diabetes, ${ }^{16,20}$ having had a stroke, ${ }^{18,20}$ living in a nursing home with frequent use of feeding tubes, ${ }^{15}$ and receiving high numbers of medications. $^{20}$

To date, there have been two studies estimating the prevalence of inappropriate prescribing in Australian nursing home residents with dementia. ${ }^{21,22}$ Bosboom et al (2012) included residents at varying stages of dementia progression and used general-elderly measures of potentially inappropriate prescribing [Beers criteria, Drug Burden Index $(>0)$ and polypharmacy ( $\geq 5$ medications)] 
rather than dementia-specific or palliative-specific criteria. Somers et al (2010) also used the Beers criteria to determine appropriateness of medications in their sample of residents with dementia.

More recently, a new set of delphi constructed guidance measures have been developed by Australian experts to help guide clinicians who manage co-morbid conditions in people with dementia. ${ }^{23}$ The authors also further externally validated these guidance statements in focus groups with health professionals to determine their utility in clinical practice. ${ }^{24}$ However, these guidance measures have not been designed to identify the prevalence of potentially inappropriate medications via chart audit.

The current project aimed to estimate the prevalence of potentially inappropriate prescribing in Australian nursing home residents with advanced dementia, using the disease- and stage-specific PEACE criteria. Specific objectives were to identify: 1) the proportion of residents taking 'never' appropriate medications, 2) the most commonly prescribed 'never' appropriate medications, and 3) factors associated with an increased likelihood of residents receiving 'never' appropriate medications.

\section{Methods}

\section{Study design}

This research formed a sub-study of a cluster randomised controlled trial (RCT) of facilitated case conferencing for residents with advanced dementia living in 20 nursing homes in Sydney and Brisbane, Australia (the 'IDEAL Study'). ${ }^{25}$ The sub-study used a retrospective medication chart audit.

\section{Ethics approval}

Approval to undertake this research was obtained from the University of New South Wales Human Research Ethics Committee (approval number HC12455). Because individuals with advanced dementia lack capacity to provide informed consent, ethics approval was gained for proxy consent by a family member. Medication data were collected from June 2013 to December 2014.

\section{Study population}

\section{Nursing homes}

Nursing homes were eligible to participate in the IDEAL Study if they were located in the greater metropolitan areas of Sydney or Brisbane and met the following criteria: 1) $\geq 100$ high care beds and 2) $\geq 50 \%$ residents with dementia (or equivalent number of residents with dementia achieved by a higher proportion or residents with dementia but lower number of beds). 
To minimise selection bias, eligible nursing homes were identified via the Aged Care Australia website (recently superseded by http://www.myagedcare.gov.au/) and approached in random order until the target sample size of 20 was achieved.

\section{Residents}

To be eligible for the IDEAL Project, residents needed to have: 1 ) dementia status reported in their progress notes and level of cognitive impairment stable for $\leq 1$ month according to residential aged care staff; 2 ) advanced dementia as determined by screening using the Functional Assessment Staging Tool $(\text { FAST })^{26}$ of $\geq 6$; and an Australia-modified Karnofsky Performance Status (AKPS) ${ }^{27}$ score of $\leq 50$; 3) availability of a person legally responsible to give consent on their behalf; and 4) informed consent from a family member or other who knew the resident well and was willing to participate in IDEAL to report their perception of quality of care. Criterion 2 was chosen because dementia stage and functioning are predictive of $<6$ months survival ${ }^{28}$ and the IDEAL Project's primary endpoint related to end-of-life care.

\section{Data collection}

\section{Socio-demographic and clinical data}

Wherever available and relevant to the Australian setting, data for the current analyses used variables found by previous studies to be significantly predictive of 'never' appropriate prescribing as defined by the PEACE criteria. ${ }^{15-18}$ These included gender ${ }^{16}$, length of stay at the nursing home,${ }^{16}$ functional status ${ }^{16}$, dementia status, ${ }^{16}$ diabetes ${ }^{16}$ and stroke. ${ }^{18}$

Significant predictors found in two studies ${ }^{19,20}$ utilising similar approaches to Holmes et al 2008 were excluded from analysis for the following reasons. Differences in the way Parsons et al (2015) categorised medications from Holmes et al include reaching consensus for aspirin, iron, vitamins, mineral supplements and finasteride, and placing them under the 'never' appropriate category. ${ }^{19}$ Parsons et al's criteria also deviated from Holmes et al by placing acetylcholinesterase inhibitors under 'rarely' appropriate and memantine under 'sometimes' appropriate, both of which were under 'never' appropriate in the PEACE criteria. We chose not to include Parsons et al as a source study because of these medication classification differences, and because they used a small sample of only 15 residents. Matlow et al 2017 focused their analysis on the last week of life, making their sample incomparable to our own.

Data on dementia stage and functional status collected at baseline were used for this sub-study's analysis and were collected using the FAST and AKPS respectively. All other data were collected from administrative and nursing records kept at each nursing home. 
The FAST divides progression of dementia into seven distinct stages based on observable cognitive and behavioural symptoms. The observational nature of the FAST enables it to be used to assess moderate-severe stages of dementia when cognitive tests such as the Mini Mental State Examination $(\mathrm{MMSE})^{29}$ no longer provide useful information.

The AKPS assigns a single score between 0 (dead) and 100 (normal; no complaints; no evidence of disease) based on observation of functional independence. A score of 50 , the maximum at which a resident was deemed eligible for the current study, indicates that a person 'requires considerable assistance and frequent medical care'.

Resident variables found to be predictive of 'never' appropriate prescribing by previous studies but either not relevant to the Australian setting or unavailable in the IDEAL Study dataset were AfricanAmerican ethnicity, ${ }^{15}$ and hospitalizations in the last 90 days ${ }^{15}$ respectively. Use of feeding tubes was found predictive in one previous study, ${ }^{15}$ however none of the residents participating in the IDEAL study was using a feeding tube. Nursing home variables found predictive that had no variability in the IDEAL Study sample were dementia specificity, ${ }^{15}$ metropolitan status ${ }^{15}$ and facility-level use of feeding tubes; all nursing homes participating in the IDEAL Study included high proportions of residents with dementia, were metropolitan and had equally low proportions of residents using feeding tubes.

\section{Medication charts}

To increase reliability, data collection and coding was undertaken via a standardised approach maintained for all residents in all nursing homes, leaving little room for error or variation in investigator interpretation..$^{30}$ Medication charts were reviewed for each resident and names extracted as written. This sub-study focused on regularly prescribed medications because of the difficulty in deducing burden from pro re nata (PRN) and short-term medications.

Medication chart data were entered into Microsoft Excel version 10 (Microsoft Corporation, Seattle) using study numbers to identify each resident. Medications were then coded according to the Anatomical Therapeutic Chemical (ATC) Classification System, an international standard for drug utilisation studies recognised by the World Health Organisation ${ }^{31}$ and used extensively in literature to categorise medications.

Once ATC codes had been allocated to each medication, these were then classified according to PEACE criteria for 'never' appropriate prescribing, adopting the codes reported by Toscani et al $(2013)^{17}$ (see Box 1). 
Antiplatelet agents, excluding Aspirin (B01AC excl B01AC06); Lipid-lowering medications (C10); Sex hormones (G03H); Cytotoxic chemotherapy (L01); Hormone antagonists (L02B); Antiestrogens (L02BA; L02BB); Immunomodulators (L03); memantine (N06DX01); Acetylcholinesterase inhibitors (N07AA); Leucotriene receptor antagonists (R03DC)

Medications with ATC code N06D were included to this study's classification of 'never' appropriate acetylcholinesterase inhibitors.

While we used the same codes previously selected by Toscani et al, there was one exception where they included N06D acetylcholinesterase inhibitors into the 'always' appropriate category, possibly due to the recent evidence showing effectiveness in severe dementia. However, we chose to include these medications under the 'never' appropriate category, as the safety and benefit of these medications for individuals receiving palliative care is still contentious.

\section{Statistical analyses}

Descriptive analyses were conducted to provide summary characteristics for residents. A chi-square analysis was conducted to test whether there was a significant difference in the percentages of residents taking a 'never' appropriate medication between intervention and control groups. Where no significant difference was observed, it was considered appropriate to combine the arms for all further analyses.

Generalized Linear Mixed Models (GLMM) with a logit link function were used to determine predictors of receiving a 'never' appropriate medication (binomial distribution). GLMMs allow for the inclusion of fixed and random effects in the model, and allow for nested sources of variability in data. In these models, the nursing home was included as a random effect, to account for the intra clustercorrelations in the sample and produce better fixed-effect estimates.

A preliminary GLMM included all variables found in previous studies as predictors of residents with advanced dementia receiving a 'never' appropriate medication; these variables included gender, length of stay at the nursing home (categorised into quintiles), functional status (AKPS score), dementia status (FAST score 7 vs 6 ), diabetes and stroke. The final model included only factors significant $(p<0.05)$ or approaching significance $(p<0.2)$ in the first analysis. For each model, $p$-values were provided for each estimate of fixed effects, and odds ratio (OR) and $95 \%$ confidence interval $(95 \% \mathrm{Cl})$ were provided for each fixed coefficient.

All analyses were conducted using SPSS (version 24) and $p$-values $<0.05$ considered to indicate statistical significance. 


\section{Results}

The IDEAL project recruited 13 nursing homes in Sydney and 7 in Brisbane. Nursing homes had a median of 115 beds (IQR $100-135$ ), and 12 were private and 8 not for profit. Of the 286 residents recruited to the IDEAL project, we were able to collect not only complete medication data but also socio-demographic and clinical data for 218 residents, 110 residents from intervention nursing homes and 108 from nursing homes allocated to the control group. See Table 2 for sample characteristics. A chi-square analysis found no significant difference in the percentages of residents taking a 'never' appropriate medication between intervention and control groups $(p=0.183)$, so the groups were combined for all further analyses.

Table 1. Characteristics for 218 residents with advanced dementia

\begin{tabular}{|c|c|c|c|}
\hline Characteristics & $\begin{array}{l}\text { Whole sample } \\
\qquad(\mathrm{n}=\mathbf{2 1 8})\end{array}$ & $\begin{array}{l}\text { At least one } \\
\text { 'never' } \\
\text { appropriate } \\
\text { medication } \\
(n=65) \\
\end{array}$ & $\begin{array}{l}\text { All medications } \\
\text { appropriate } \\
(n=153)\end{array}$ \\
\hline & $n(\%)$ & n (\%) & n (\%) \\
\hline Gender, female & $135(61.9)$ & $34(52.3)$ & $101(66.0)$ \\
\hline Length of stay at nursing home & C & & \\
\hline$\leq 10$ months & $47(21.6)$ & $21(32.3)$ & $26(17.0)$ \\
\hline $11-21$ months & $43(19.7)$ & $18(27.7)$ & $25(16.3)$ \\
\hline 22-37 months & $44(20.2)$ & $11(16.9)$ & $33(21.6)$ \\
\hline $38-60$ months & $41(18.8)$ & $10(15.4)$ & $31(20.3)$ \\
\hline$>5$ years & $43(19.7)$ & $5(7.7)$ & $38(24.8)$ \\
\hline \multicolumn{4}{|l|}{ AKPS } \\
\hline 20 (totally bedfast) & $40(18.3)$ & $6(9.2)$ & $34(22.2)$ \\
\hline s. & $42(19.3)$ & $13(20.0)$ & $29(19.0)$ \\
\hline 40 & $49(22.5)$ & $13(20.0)$ & $36(23.5)$ \\
\hline 50 (considerable assistance) & 87 (39.9) & $33(50.8)$ & $54(35.3)$ \\
\hline \multicolumn{4}{|l|}{ FAST } \\
\hline 6 (severe cognitive decline) & $51(23.4)$ & $17(26.2)$ & $34(22.2)$ \\
\hline 7 (very severe cognitive decline) & $167(76.6)$ & $48(73.8)$ & $119(77.8)$ \\
\hline \multicolumn{4}{|l|}{ Comorbidities } \\
\hline Stroke & $27(12.4)$ & $6(9.2)$ & $21(13.7)$ \\
\hline Diabetes & $29(13.3)$ & $12(18.5)$ & $17(11.1)$ \\
\hline
\end{tabular}

AKPS, Australia-modified Karnofsky Performance Status; ${ }^{27}$ FAST, Functional Assessment Staging Tool $^{26}$ 


\section{Prevalence of different medications}

Residents with advanced dementia were receiving an average of 7.3 (median 7.0, SD 3.5, range 0 - 21) regularly prescribed medications each. The most commonly prescribed medications types overall were laxatives (64.7\%, $n=141 / 218)$, simple analgesics (58.3\%, $n=127 / 218)$, vitamins (51.8\%, $n=113 / 218$ ) and antipsychotics (46.8\%, $n=102 / 218)$. Out of the 218 residents included in the study, 65 (29.8\%) were receiving at least one medication rated 'never' appropriate by the PEACE criteria, with $11(5.0 \%)$ receiving more than one. The most commonly prescribed 'never' appropriate medications were lipid-lowering agents, antiplatelet agents and acetylcholinesterase inhibitors (see Table 2).

Table 2. Summary of residents receiving 'never' appropriate medications, $\mathbf{N}=\mathbf{2 1 8}$ residents

\begin{tabular}{|l|l|}
\hline Medication type & $\begin{array}{l}\text { Number of residents receiving (\% of total } \\
\text { residents) }\end{array}$ \\
\hline Lipid-lowering medications & $\mathbf{n}(\%)$ \\
\hline Antiplatelet agents & $38(17.4)$ \\
\hline Acetylcholinesterase inhibitors & $18(8.3)$ \\
\hline Memantine & $16(7.3)$ \\
\hline Cytotoxic chemotherapy & $6(2.8)$ \\
\hline Antiestrogens & $1(0.5)$ \\
\hline Hormone antagonists & $1(0.5)$ \\
\hline
\end{tabular}

\section{Predictors of 'never' appropriate medications}

Of factors associated with 'never' appropriate medications in previous studies, only shorter length of stay was found predictive in the current sample $(F(4,206)=2.61, p=0.037$; Table 3$)$. Residents who had been at the nursing home less than or equal to 10 months, or between 11 and 21 months, had 5 times greater odds of receiving a 'never' appropriate medication than residents who had been at the nursing home for $61+$ months ( $>5$ years).

While functional status (AKPS) as an overall fixed effect was not found to be significant $(F(3,206)=$ 1.75, $p=0.16)$, residents with AKPS 20 had significantly lower odds of receiving a 'never' appropriate medication compared to those with AKPS 50 (OR 0.33, 95\% CI 0.11-0.98; see Table 3). 


\begin{tabular}{|c|c|c|c|c|}
\hline Variable & Categories & OR & $95 \% \mathrm{Cl}$ & P Value \\
\hline \multirow[t]{2}{*}{ Sex } & Male & 1.102 & $0.729,2.813$ & 0.295 \\
\hline & Female & - & & \\
\hline \multirow{6}{*}{$\begin{array}{l}\text { Length of } \\
\text { Stay }\end{array}$} & & & & 0.037 \\
\hline & $\leq 10$ months & 5.349 & $1.600,17.879$ & 0.007 \\
\hline & $11-21$ months & 5.624 & $1.673,18.911$ & 0.005 \\
\hline & 22-37 months & 2.866 & $0.826,9.937$ & 0.097 \\
\hline & $38-60$ months & 2.459 & $0.705,8.582$ & 0.157 \\
\hline & $>5$ years & - & & 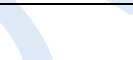 \\
\hline \multirow[t]{5}{*}{ AKPS } & & & & 0.158 \\
\hline & 20 & 0.329 & $0.110,0.980$ & 0.046 \\
\hline & 30 & 1.088 & $0.423,2.797$ & 0.861 \\
\hline & 40 & 0.625 & $0.253,1.545$ & 0.308 \\
\hline & 50 & & & \\
\hline \multirow[t]{2}{*}{ FAST } & Score 6 & 1.015 & $0.296,1.483$ & 0.315 \\
\hline & Score 7 & - & & \\
\hline \multirow[t]{2}{*}{ Stroke } & No & 1.218 & $0.418,3.547$ & 0.716 \\
\hline & Yes & - & & \\
\hline \multirow[t]{2}{*}{ Diabetes } & No & 0.482 & $0.191,1.214$ & 0.121 \\
\hline & Yes & - & & \\
\hline
\end{tabular}

AKPS, Australia-modified Karnofsky Performance Status ${ }^{27}$ (lower scores indicate greater assistance required); FAST, Functional Assessment Staging Tool $^{26}$ (higher scores indicate greater functional deterioration); $\mathrm{Cl}$, confidence interval; $\mathrm{OR}$, odds ratio. Reference categories indicated by ' - . The $\mathrm{p}$ values correspond to the fixed effects $F$ statistics. The results are based on the generalized linear mixed effect model (GLMM) analysis with nursing home as a random effect. Statistically significant odds ratios are in bold $(\mathrm{P}<0.05)$

Table 4 shows the results of the final GLMM model which included length of stay, functional status (AKPS) and diabetes. Length of stay continued to be the only significant predictor of receiving a 'never' appropriate medication $(F(4,209)=2.84, p=0.025)$. 
Table 4: Final model of associations of potential risk of receiving 'never' appropriate medications $(\mathrm{N}=218)$

\begin{tabular}{|c|c|c|c|c|}
\hline Variable & Categories & OR & $95 \% \mathrm{Cl}$ & P Value \\
\hline \multirow{6}{*}{$\begin{array}{l}\text { Length of } \\
\text { Stay }\end{array}$} & & & & 0.025 \\
\hline & $\leq 10$ months & 5.599 & $1.736,18.055$ & 0.004 \\
\hline & $11-21$ months & 5.411 & $1.666,17.750$ & 0.005 \\
\hline & 22-37 months & 2.836 & $0.838,9.592$ & 0.093 \\
\hline & $38-60$ months & 2.415 & $0.708,8.242$ & 0.158 \\
\hline & $>5$ years & - & & \\
\hline \multirow[t]{5}{*}{ AKPS } & & & & 0.221 \\
\hline & 20 & 0.375 & $0.132,1.067$ & 0.066 \\
\hline & 30 & 1.101 & $0.454,2.667$ & 0.831 \\
\hline & 40 & 0.662 & $0.285,1.538$ & 0.335 \\
\hline & 50 & - & & \\
\hline \multirow[t]{2}{*}{ Diabetes } & No & 0.445 & $0.181,1.093$ & 0.077 \\
\hline & Yes & - & & \\
\hline
\end{tabular}

AKPS, Australia-modified Karnofsky Performance Status ${ }^{27}$ (lower scores indicate greater assistance required); FAST, Functional Assessment Staging Tool $^{26}$ (higher scores indicate greater functional deterioration); $\mathrm{Cl}$, confidence interval; $\mathrm{OR}$, odds ratio. The $\mathrm{p}$-values correspond to the fixed effects $\mathrm{F}$ statistics. The results are based on the generalized linear mixed effect model (GLMM) analysis with nursing home as a random effect. Statistically significant odds ratios are in bold $(P<0.05)$

\section{Discussion}

This study is the first to estimate prevalence of potentially inappropriate prescribing in Australian nursing home residents with advanced dementia using the PEACE criteria, an explicit disease- and stage-specific criteria. Nursing home residents in the current study were taking an average of seven medications, with nearly one third receiving at least one medication rated 'never' appropriate. Similar to previous studies, lipid-lowering agents, ${ }^{15,16,18}$ antiplatelet agents ${ }^{15,17,18}$ and acetylcholinesterase inhibitors ${ }^{15,16,18}$ were the most commonly prescribed 'never' appropriate medications. Our data also replicate previous findings that a shorter length of stay ${ }^{16,18}$ is a significant predictor for receiving one or more 'never' appropriate medications, highlighting the need for medication review shortly after a resident's admission to ensure medications are consistent with a palliative approach where 
appropriate.Two studies have identified an association between inappropriate medication use and higher functional status. ${ }^{15,32}$ While our study did not find an overall association between functional status (AKPS score) and receiving 'never' appropriate medications, our results trended towards the same direction, with residents who had a lower functional status (AKPS 20, totally bedfast) being significantly less likely than those with the highest functional status (AKPS 50, requiring considerable assistance and frequent medical care) to receive a 'never' appropriate medication.

The finding that a shorter length of stay increases the odds of receiving 'never' appropriate medications in residents with advanced dementia may suggest that a longer stay allows for more time for conversations to be had with substitute decision makers regarding goals of care, and a rationale developed for stopping medications where applicable. This finding may also highlight the need for medication review early on or even at the time of a resident's admission to ensure medications are consistent with a palliative approach.

No significant change in odds of receiving inappropriate medications between residents with higher and lower cognitive and functional status is troubling as it suggests recommended monitoring, review and deprescribing of unnecessary medications as end of life approaches may not yet be a common practice of medication management in residents with advanced dementia.

The proportion of residents (29.8\%) taking 'never' appropriate drugs in the current study was lower than those in similar studies undertaken in the US $(37 \%, 54 \%),{ }^{15}, 16$ similar to that found in a panEuropean study (27\%), ${ }^{18}$ and higher than that reported in a study exclusively undertaken in Italy (3\%). ${ }^{17}$ These inconsistencies may be due to differences in prescribing cultures between countries or differences in the ways studies sampled nursing homes and residents.

Rationalising the use of 'never' appropriate medications like lipid-lowering agents and antiplatelet agents is difficult. Their therapeutic goal to reduce vascular events and mortality becomes irrelevant in a population where maintaining comfort rather than extending life is the focus, and time to benefit almost certainly exceeds the person's life span. Both pose risks of adverse effects, including abdominal pain, constipation and nausea for lipid-lowering agents, ${ }^{33,34}$ and haemorrhage of the gastrointestinal tract for antiplatelet agents..$^{35}$ Australian clinical practice guidelines ${ }^{36,37}$ provide recommendations on the rational use and deprescribing acetylcholinesterase inhibitors and memantine. While these medications may be appropriate for people with advanced dementia under some circumstances, evidence of their benefits is limited ${ }^{38,39}$ and has been met with criticism. ${ }^{40}$ They also have side-effects including nausea, vomiting, diarrhoea and muscle cramps. ${ }^{41}$ 
The high rates of residents prescribed antipsychotics (47\%) is much higher than previously reported in studies in other countries ${ }^{15-17}$ which range from $27 \%{ }^{16}$ to $30 \% .{ }^{17}$ Whilst classed as 'sometimes' rather than 'never' appropriate by the PEACE criteria, a report by Alzheimer's Australia (2014) recommended the use of antipsychotics should be time limited and reviewed regularly with multidisciplinary input from pharmacists, behaviour management experts, GPs and psychiatrists. ${ }^{32}$

As well as identifying high rates of medicines of concern, our study found that laxatives $(65 \%)$ and simple analgesics (58\%) were the most commonly prescribed medications overall. Rates of analgesic use were similar to results from US studies $\left(58 \%{ }^{16}\right.$ and $\left.59 \%{ }^{15}\right)$ and substantially higher than rates in the Italian study $\left(8.1 \%^{17}\right)$. While our analysis did not examine the reasons for prescription, higher rates of analgesic and laxative use are generally encouraging given evidence that symptoms often go unrecognised and under-treated in this population. ${ }^{42-44}$

\section{Limitations}

The limitations of this study arise from data being collected via retrospective chart audit, its dependence on a single method (the PEACE criteria) of identifying inappropriate prescribing, and its status as an RCT sub-study. Problems relating to retrospective chart audits identified in the literature include a lack of standardisation in chart formats utilised, incomplete records and illegibility. ${ }^{45}$ The current study sought to minimise these problems by using residents' medication chart as the primary source of data - charts that are required to be up-to-date and legible for regular use and surveillance. However, an exclusive focus on medication charts meant the study lacked information on the reasons 'never' appropriate medications had been prescribed and had not been withdrawn. Even a detailed review of progress notes and clinical assessments would have been unlikely to inform understanding of appropriateness for each resident given variability in the quality of reporting and long duration of prescription in many cases, for some residents even prior to admission. The PEACE criteria categories offer a 'one size fits all' approach to identifying inappropriate medications and do not take into account clinical factors and context that may be at play in individual cases. Holmes et al (2008) identified a need for future research focusing on larger sample populations which can better showcase the relationship between comorbidities and medication use, as well as identify distinct medication classes that may be overused ${ }^{14}$. Dependence on IDEAL Study data meant that some variables found predictive by previous studies (especially those at the nursing home level) could not be included in the current analyses. Also, the nursing homes involved in the RCT may have been more aware of issues relating to inappropriate prescribing than the industry average. Twenty two percent of residents in the intervention arm ( $11 \%$ of the total sample) had their medication charts collected after the intervention commenced at their nursing home, raising the possibility that the intervention may have 
influenced prescribing, even though this was not of a magnitude that led to a significant difference in the number of residents taking 'never' appropriate medications in each arm. These factors mean that our results may have underestimated the prevalence of inappropriate prescribing in Australian nursing homes more generally. Finally, our study shares a limitation with those previous in not exploring the risk and seriousness of adverse effects from being prescribed a never appropriate medication. More research is needed to establish the clinical outcomes of such prescriptions in nursing home residents with advanced dementia.

\section{Conclusion}

To our knowledge, this is the first Australian study to estimate the prevalence of inappropriate prescribing in nursing home residents with advanced dementia using a disease- and stage- specific tool. Our results indicate that a significant minority of residents may be taking inappropriate medications including lipid-lowering medications, antiplatelet agents and acetylcholinesterase inhibitors. These findings are limited by use of only one set of criteria for identifying inappropriate medications and the lack of detailed clinical data to contextualise prescribing for each individual resident. More research is needed to guide deprescribing of these medicines, and the associated negative outcomes of their use in advanced dementia.

\section{Acknowledgments}

We would like to acknowledge the IDEAL Study project team for their assistance in collecting the data in this sub-study, including Janet Cook, Deborah Brooks, Priyanka Bhattarai, Huali (Molly) Cao and Dr Jennifer Houltram.

\section{Author Disclosure Statement}

The IDEAL Study was funded by the Australian Department of Health (previously Department of Health and Ageing). DD was provided a PhD scholarship from the University of Technology Sydney. All other authors have no potential conflicts of interest that are directly relevant to the content of this study.

\section{Authors' Contribution}

$\mathrm{DD}, \mathrm{TL}, \mathrm{PMD}, \mathrm{SB}$ and MA contributed to design of the research. DD carried out the data acquisition and analysis. GL advised on statistical analysis. TL and MA were involved in interpretation of results. All authors contributed to writing the report and approved the final manuscript. 


\section{References}

1. Mitchell SL, Teno JM, Kiely DK, Shaffer ML, Jones RN, Prigerson HG, et al. The clinical course of advanced dementia. New England Journal of Medicine. 2009;361(16):1529-38.

2. Agar M, Beattie E, Luckett T, Phillips J, Luscombe G, Goodall S, et al. Pragmatic cluster randomised controlled trial of facilitated family case conferencing compared with usual care for improving end of life care and outcomes in nursing home residents with advanced dementia and their families: the IDEAL study protocol. BMC palliative care. 2015;14(1):63.

3. Stevenson J, Abernethy AP, Miller C, Currow DC. Managing comorbidities in patients at the end of life. BMJ. 2004;329(7471):909-12.

4. Budnitz DS, Lovegrove MC, Shehab N, Richards CL. Emergency hospitalizations for adverse drug events in older Americans. New England Journal of Medicine. 2011;365(21):2002-12.

5. Kalisch LM, Caughey GE, Barratt JD, Ramsay EN, Killer G, Gilbert AL, et al. Prevalence of preventable medication-related hospitalizations in Australia: an opportunity to reduce harm. International Journal for Quality in Health Care. 2012;24(3):239-49.

6. Cahir C, Fahey T, Teeling M, Teljeur C, Feely J, Bennett K. Potentially inappropriate prescribing and cost outcomes for older people: a national population study. Br J Clin Pharmacol. 2010;69(5):543-52.

7. Hovstadius B, Petersson $G$. The impact of increasing polypharmacy on prescribed drug expenditure-a register-based study in Sweden 2005-2009. Health Policy. 2013;109(2):166-74.

8. Parsons C, Hughes CM, Passmore AP, Lapane KL. Withholding, discontinuing and withdrawing medications in dementia patients at the end of life: a neglected problem in the disadvantaged dying? Drugs Aging. 2010;27(6):435-49.

9. Lau DT, Mercaldo ND, Harris AT, Trittschuh E, Shega J, Weintraub S. Polypharmacy and potentially inappropriate medication use among community-dwelling elders with dementia. Alzheimer Dis Assoc Disord. 2010;24(1):56-63.

10. Riker GI, Setter SM. Polypharmacy in older adults at home: what it is and what to do about it--implications for home healthcare and hospice. Home Healthc Nurse. 2012;30(8):474-85; quiz 867.

11. Beers MH, Ouslander JG, Rollingher I, Reuben DB, Brooks J, Beck JC. Explicit criteria for determining inappropriate medication use in nursing home residents. UCLA Division of Geriatric Medicine. Archives of Internal Medicine. 1991;151(9):1825-32.

12. Fick DM, Cooper JW, Wade WE, Waller JL, Maclean JR, Beers MH. Updating the Beers criteria for potentially inappropriate medication use in older adults: results of a US consensus panel of experts. Archives of Internal Medicine. 2003;163(22):2716.

13. Holmes HM, Sachs GA, Shega JW, Hougham GW, Cox Hayley D, Dale W. Integrating palliative medicine into the care of persons with advanced dementia: identifying appropriate medication use. Journal of the American Geriatrics Society. 2008;56(7):1306-11.

14. Disalvo D, Luckett T, Agar M, Bennett A, Davidson PM. Systems to identify potentially inappropriate prescribing in people with advanced dementia: a systematic review. BMC geriatr. 2016;16(1):114.

15. Tjia J, Briesacher BA, Peterson D, Liu Q, Andrade SE, Mitchell SL. Use of medications of questionable benefit in advanced dementia. JAMA internal medicine. 2014;174(11):1763-71.

16. Tjia J, Rothman MR, Kiely DK, Shaffer ML, Holmes HM, Sachs GA, et al. Daily medication use in nursing home residents with advanced dementia. Journal of the American Geriatrics Society. 2010;58(5):880-8.

17. Toscani F, Di Giulio P, Villani D, Giunco F, Brunelli C, Gentile S, et al. Treatments and prescriptions in advanced dementia patients residing in long-term care institutions and at home. Journal of Palliative Medicine. 2013;16(1):31-7.

18. Colloca G, Tosato M, Vetrano DL, Topinkova E, Fialova D, Gindin J, et al. Inappropriate drugs in elderly patients with severe cognitive impairment: results from the shelter study. PloS one.

2012;7(10):e46669. 
19. Parsons C, McCann L, Passmore P, Hughes C. Development and application of medication appropriateness indicators for persons with advanced dementia: a feasibility study. Drugs \& aging. 2015;32(1):67-77.

20. Matlow JN, Bronskill SE, Gruneir A, Bell CM, Stall NM, Herrmann N, et al. Use of Medications of Questionable Benefit at the End of Life in Nursing Home Residents with Advanced Dementia. Journal of the American Geriatrics Society. 2017.

21. Bosboom PR, Alfonso H, Almeida OP, Beer C. Use of potentially harmful medications and health-related quality of life among people with dementia living in residential aged care facilities. Dementia and geriatric cognitive disorders extra. 2012;2(1):361-71.

22. Somers M, Rose E, Simmonds D, Whitelaw C, Calver J, Beer C. Quality use of medicines in residential aged care. Aust Fam Physician. 2010;39(6):413-6.

23. Page AT, Clifford RM, Potter K, Schwartz D, Etherton-Beer CD. The feasibility and effect of deprescribing in older adults on mortality and health: a systematic review and meta-analysis. $\mathrm{Br} \mathrm{J}$ Clin Pharmacol. 2016;82(3):583-623.

24. Page AT, Clifford RM, Potter K, Seubert L, McLachlan AJ, Hill X, et al. Exploring the enablers and barriers to implementing the Medication Appropriateness Tool for Comorbid Health conditions during Dementia (MATCH-D) criteria in Australia: a qualitative study. BMJ open. 2017;7(8):e017906. 25. Agar M, Luckett T, Luscombe G, Phillips J, Beattie E, Pond D, et al. Effects of facilitated family case conferencing for advanced dementia: A cluster randomised clinical trial. PLOS ONE. 2017;12(8):e0181020.

26. Reisberg B. Functional Assessment Staging (FAST). Psychopharmacology Bulletin 1988. 1988;24:653-9.

27. Abernethy AP, Shelby-James T, Fazekas BS, Woods D, Currow DC. The Australia-modified Karnofsky Performance Status (AKPS) scale: a revised scale for contemporary palliative care clinical practice [ISRCTN81117481]. BMC palliative care. 2005;4(1):7.

28. Coventry PA, Grande GE, Richards DA, Todd CJ. Prediction of appropriate timing of palliative care for older adults with non-malignant life-threatening disease: a systematic review. Age Ageing. 2005;34(3):218-27.

29. Folstein MF, Folstein SE, McHugh PR. "Mini-mental state". A practical method for grading the cognitive state of patients for the clinician. Journal of Psychiatric Research. 1975;12(3):189-98. 30. Grimes DA, Schulz KF. Bias and causal associations in observational research. The Lancet. 2002;359(9302):248-52.

31. WHO Collaborating Centre for Drug Statistics Methodology. Guidelines for ATC Classification and DDD Assignment 2013. 2013.

32. Alzheimer's Australia. The Use of Restraints and Psychotropic Medications in People with Dementia: A Report For Alzheimer's Australia. 2014.

33. Hilmer S, Gnjidic D. Statins in older adults. Australian Prescriber. 2013.

34. Armitage J. The safety of statins in clinical practice. The Lancet. 2007;370(9601):1781-90.

35. Salvi F, Marchetti A, D'Angelo F, Boemi M, Lattanzio F, Cherubini A. Adverse drug events as a cause of hospitalization in older adults. Drug Saf. 2012;35(1):29-45.

36. Clinical Practice Guidelines for Dementia in Australia. 2015.

37. Reeve E, Farrell B, Thompson W, Herrmann N, Sketris I, Magin P, et al. Evidence-based Clinical Practice Guideline for Deprescribing Cholinesterase Inhibitors and Memantine in People with Dementia.

38. Winblad B, Kilander L, Eriksson S, Minthon L, Båtsman S, Wetterholm A-L, et al. Donepezil in patients with severe Alzheimer's disease: double-blind, parallel-group, placebo-controlled study. The Lancet. 2006;367(9516):1057-65.

39. Howard R, McShane R, Lindesay J, Ritchie C, Baldwin A, Barber R, et al. Donepezil and memantine for moderate-to-severe Alzheimer's disease. New England Journal of Medicine. 2012;366(10):893-903. 
40. Suzuki T, Howard R, McShane R, Lindesay J. Discontinuing Donepezil or Starting Memantine for Alzheimer's Disease. The New England journal of medicine. 2012;366(23):2227.

41. Rogers SL, Friedhoff LT. The efficacy and safety of donepezil in patients with Alzheimer's disease: results of a US multicentre, randomized, double-blind, placebo-controlled trial. Dementia and Geriatric Cognitive Disorders. 1996;7(6):293-303.

42. Bayer A. Death with dementia-the need for better care. Age and Ageing. 2006;35(2):101-2. 43. Chang E, Daly J, Johnson A, Harrison K, Easterbrook S, Bidewell J, et al. Challenges for professional care of advanced dementia. International Journal of Nursing Practice. 2009;15(1):41-7. 44. McAuliffe L, Nay R, O'Donnell M, Fetherstonhaugh D. Pain assessment in older people with dementia: literature review. Journal of Advanced Nursing. 2009;65(1):2-10.

45. Jansen AC, van Aalst-Cohen ES, Hutten BA, Büller HR, Kastelein JJ, Prins MH. Guidelines were developed for data collection from medical records for use in retrospective analyses. Journal of clinical epidemiology. 2005;58(3):269-74. 